\title{
Sensory over-responsivity and ADHD: differentiating using electrodermal responses, cortisol, and anxiety
}

\author{
Shelly J. Lane ${ }^{1}{ }^{*}$, Stacey Reynolds ${ }^{1}$ and Leroy Thacker ${ }^{2}$ \\ Department of Occupational Therapy, School of Allied Health Professions, Virginia Commonwealth University, Richmond, VA, USA \\ 2 Department of Biostatistics, School of Medicine, Virginia Commonwealth University, Richmond, VA, USA
}

\author{
Edited by: \\ John J. Foxe, Nathan S. Kline Institute \\ for Psychiatric Research, USA \\ City College of the City University of \\ New York, USA \\ Reviewed by: \\ Hilary Gomes, City College of New \\ York, USA \\ ${ }^{*}$ Correspondence: \\ Shelly J. Lane, Department of \\ Occupational Therapy, School of Allied \\ Health Professions, Virginia \\ Commonwealth University, 730 East \\ Broad Street, Suite 2050, PO Box \\ 980008, Richmond, VA 23298-0008, \\ USA. \\ e-mail: sjlane@vcu.edu
}

\begin{abstract}
Deficits in sensory modulation have been linked clinically with impaired attention, arousal, and impulsivity for years, but a clear understanding of the relationship between sensory modulation disorders and attention deficit hyperactivity disorder (ADHD) has proven elusive. Our preliminary work suggested that patterns of salivary cortisol and electrodermal responsivity to sensation may be linked to different groups of children with ADHD; those with and without sensory overresponsivity (SOR). Additionally, SOR has been linked to anxiety, and anxiety has been linked to ADHD. A clearer understanding of the relationship between anxiety, SOR, and ADHD may support a better understanding of ADHD diagnostic subtypes. We examined neuroendocrine, electrodermal and behavioral characteristics and sought to predict group membership among 6to 12-year-old children with ADHD and SOR (ADHDs), ADHD and no SOR (ADHDt), and typicals (TYP). Behavioral questionnaires were completed to document SOR and anxiety. Lab testing used a Sensory Challenge Protocol (SCP) with concurrent electrodermal measurement and the collection of cortisol prior to and following the SCP. Results substantiated links between SOR and anxiety, in both TYP and ADHD children. Results suggests that ADHD should be considered in conjunction with anxiety and sensory responsivity; both may be related to bottom-up processing differences, and deficits in prefrontal cortex/hippocampal synaptic gating.
\end{abstract}

Keywords: EDR, cortisol, ADHD, sensory over-responsivity, anxiety

\section{INTRODUCTION}

Deficits in sensory modulation have been linked clinically with impaired attention, arousal, and impulsivity since the early 1970 s (Ayres, 1972). However, understanding the central nervous system processes linking sensory modulation disorders (SMD) and attention deficit hyperactivity disorder (ADHD) has proven challenging. Moving toward the goals of better understanding and improved diagnostic accuracy within and between diagnoses, investigators have used parent report measures of children's behavior, neurophysiologic measures, and neuroendocrine measures of stress responses. However, in order to distinguish SMD from ADHD, and/or clarify the relationship between the two, a more thorough understanding of the central nervous system processes as well as behavioral symptomatology within and between the disorders is necessary.

Sensory over-responsivity (SOR) is a type of SMD characterized by responses to sensory stimuli that are faster, longer, or more intense than what would be expected with typical sensory responsivity (Miller et al., 2007). Individuals may demonstrate over-responsivity to any type of sensory stimuli (e.g., sound, movement, or touch) and behavioral responses in the face of adverse stimuli. Responses may include aggression, fear, avoidance, withdrawal, irritability, or moodiness. SOR independent of other diagnoses is just beginning to gain recognition. Kinnealey and colleagues (Kinnealey et al., 1995; Kinnealey and Fuiek, 1999) documented SOR as an independent disorder in adults; Reynolds and Lane (2007) described SOR independent of other diagnoses in three children. Recent evidence indicates that gifted children may also show sensory sensitivities (Gere et al., 2009). Initial work in our lab (Reynolds et al., 2009) used a Sensory Challenge Protocol (SCP) and measured changes in salivary cortisol as a reflection of changes in the hypothalamic-pituitary-adrenal (HPA) axis, and, as suggested by other investigators, a physiologic reflection of the stress response (Schmidt, 1997; Hanrahan et al., 2006). Our preliminary findings had indicated different patterns of cortisol response to sensory challenge in children with ADHD and SOR (ADHDs), compared to that seen in children with ADHD but no SOR (ADHDt) and typical children (TYP). Children with ADHDt showed the expected blunting of the cortisol response; children with ADHDs did not. Other investigators have also indicated that a blunted cortisol response to stressful situations in children with ADHD was commonly, although not invariably, seen (King et al., 1998; Kariyawasam et al., 2002; Hong et al., 2003). Why some children with ADHD have a blunted response while others do not has to this point not been well explained; examining cortisol relative to sensory modulation may provide needed clarification.

Parush et al. (2007) also examined CNS functions in children with $A D H D$ and SOR. These investigators found that children with ADHD and tactile SOR had different central processing of somatosensory input, measured via EEG recordings, than children with ADHD without tactile SOR. The work of both Parush et al. and Reynolds et al. support the notion that SOR may be a pre-existing variable of interest in the creation of ADHD subtypes (with and without SOR), and that neurophysiological functioning may be used as biomarkers in group differentiation. 
Another way to examine diagnostic connections between ADHD and SOR is through links with anxiety. Anxiety is a comorbid condition associated with $25-33 \%$ of children with $\mathrm{ADHD}$; higher rates have been noted in children with $\mathrm{ADHD}$ and co-morbid disruptive behavior disorders such as conduct disorder (CD) or oppositional defiant disorder (ODD) (Pliszka, 1998; Jensen et al., 2001; Schatz and Rostain, 2006). In addition to behaviors such as impulsivity and fearlessness that tend to characterize children with ADHD, these children may also manifest behaviors such as moodiness, excessive worry, trouble shifting attention, and inflexibility. These characteristics overlap with those of anxiety disorders (Levy, 2004). It is unclear what causes anxiety in children, and why some, but not all, children with ADHD develop patterns of anxiety.

It has long been theorized that anxiety is the result of faulty information processing, as well as a hypersensitivity to information and stimuli in the environment (Ayres, 1972; Johnson, 1975). Ayres further suggested that deficits in the ability to modulate incoming sensory stimuli lead to the manifestation of distractibility, anxiety, and other stress related behaviors. Research in both pediatric and adult populations has produced preliminary evidence of links between SOR and anxiety. Pfeiffer et al. (2005) administered the Sensory Profile (Dunn, 1999) or the Adolescent/Adult Sensory Profile (Brown and Dunn, 2002) to 46 subjects, ages 6-16, diagnosed with Asperger's syndrome. Using the Revised Children's Manifest Anxiety Scale (Reynolds and Richmond, 2005), they found a significant positive relationship between SOR and anxiety for the entire group of children. Using the same anxiety scale, Reynolds and Lane (2009) documented a link between SOR and anxiety, in children with ADHD. Neal et al. (2002) also found a positive relationship between self-reported anxiety and sensitivity to environmental stimuli in adults ages $17-75$. In this study scores from the Highly Sensitive Person Scale (Aron and Aron, 1997) were found to significantly predict anxiety in individuals classified with agoraphobia, anxiety/panic, and social phobia (Neal et al., 2002).

These investigations indicate that sensory over-responsiveness should be a consideration for children with ADHD; that it may distinguish between subgroups of children with ADHD; and that it is seen in the absence of ADHD. Further, SOR and anxiety have been linked, and anxiety and ADHD have been shown to co-exist. The current study investigated children with and without ADHD, examining the responses to sensory challenge. Our prior work had supported a link between cortisol, anxiety, and SOR; goals for this study were twofold: (1) determine if children with ADHD and SOR (ADHDs) could be differentiated from children with ADHD but no SOR (ADHDt), typical children (TYP), and typical children with SOR (TYPs) based on neuroendocrine (salivary cortisol) and electrodermal markers; (2) assess whether a combination of anxiety, physiological and neuroendocrine variables could be used to predict membership in one of the groups (TYP, TYPs, ADHDs, ADHDt).

\section{MATERIALS AND METHODS}

This study was approved by the sponsoring university's Institutional Review Board prior to beginning subject recruitment. The overall research design requested parents and children to complete forms reflecting sensory processing and perceived anxiety prior to lab testing. Lab testing used the SCP, the collection of salivary cortisol prior to and following the SCP, and the collection of electrodermal response (EDR) data during the SCP.

A convenience sample $(n=84)$ between the ages of 6 and 12 were enrolled in this study. Thirty-nine children with a diagnosis of ADHD were recruited under the guidance of the university's Department of Psychiatry division chair, who verified the diagnosis of ADHD through clinical evaluation and/or record review. A control group $(n=46)$ without ADHD was recruited through informational flyers posted on medical and academic campuses of the sponsoring institution and at recreation centers in the metropolitan area. Children were considered typical if they had no diagnosis or behavioral concerns. Children with psychological diagnoses other than ADHD, significant motor impairments such as cerebral palsy, or any known endocrine or metabolic dysfunctions were excluded from this study. All children were screened for normal intelligence (IQ > 70) using a two subtest battery of the Wechsler Abbreviated Scale of Intelligence (WASI) (PsychCorp, 1999).

\section{PROCEDURES}

Following a telephone screening, the Sensory Over-Responsivity Inventory (SensOR) (Schoen et al., 2008), the Revised Childhood Manifest Anxiety Scale (RCMAS) (Reynolds and Richmond, 2005), the informed consent/assent, and a short form requesting demographic information such as the child's age, sex, race, and current medication regimen was mailed to the family. Parents completed the SensOR and read the RCMAS questions to their child so the child could respond. On the day of testing, parents brought their child and the completed test forms to the laboratory. Prior to physiological testing, the child completed a two subtest battery of the WASI to ensure intelligence within normal limits. No child scored $<70$ on the WASI and all children were included in the study.

Before collection of the first saliva sample, children rinsed their mouths with water, washed their hands and used the lavatory facilities if needed. After becoming comfortable in the lab, the child was invited to sit in the testing chair; at this point the first cortisol sample was taken. Five surface electrodes were applied to the child's skin; two electrodes were placed on the hand to record electrodermal activity, and three were placed across the chest to record heart rate (heart rate data not reported here). Electrode accommodation to the skin took place as the child watched a 6-min movie clip. Following the accommodation period, the second baseline sample of cortisol was taken and the SCP was begun. The SCP, designed by Miller et al. (2001) involved presentation of a six different sensory stimuli in the following order: (1) Classic Tone: Steady tone played at 78 decibels; (2) Vision: Strobe light set at 10 flashes per second; (3) Sound: Recorded fire engine played at 84 decibels; (4) Smell: Wintergreen oil moved back and forth 1 " below the child's nose; (5) Touch: A feather manually stroked from the right ear along the chin to the left ear; (6) Movement: The child's chair electronically tipped back and returned to an upright position.

Each stimulus was presented eight times with a variable 10 - to 15-s interval between stimuli. At the end of each sensory domain the child was rewarded with a sticker. Timing for the stimuli was 
monitored through a customized computer program called PsyLab, developed by and purchased from Contact Precision Instruments ${ }^{1}$. Following the SCP, seven additional samples of saliva were collected at 5-min intervals. During the post-challenge sampling the children were told to rest and watch a 30 -min silent cartoon.

\section{INSTRUMENTATION AND MEASURES Sensory over-responsivity scale}

The SensOR Inventory (Schoen et al., 2008), a caregiver-report tool, identifies individuals with SOR in one or more of six sensory domains: tactile, auditory, visual, olfactory, taste, and vestibularproprioceptive. While other scales such as the Short Sensory Profile (McIntosh et al., 1999a) identify general SMDs in children, they do not specifically identify children with over-responsiveness. The SensOR is currently the only scale that clearly differentiates SOR from typical responsivity (Schoen et al., 2008). Version 1.4 of the SensOR Inventory, used in the current study, is a 76-item questionnaire, with items falling into eight sensory categories: TactileTextures, Tactile-Activities of Daily Living, Auditory Settings, Auditory-Specific, Visual, Olfactory, Movement-Proprioceptive, and Food-Textures/Eating. For this study, items were re-grouped into tactile (tactile-textures + tactile-activities of daily living), auditory (auditory settings + auditory specific), visual, smell, taste (food-textures/eating), and movement (movement-proprioceptive) domains. Internal consistency reliability was high within each domain (Cronbach's $\alpha=0.65-0.88$ ). In addition, the SensOR Inventory was shown to have strong discriminant validity, distinguishing between individuals with and without SOR within each domain $(p<0.05-0.001)$ (Schoen et al., 2008). The SensOR also has been shown to have strong concurrent validity with the overresponsive scales on the Short Sensory Profile $(r=0.47, p<0.01)$. We have conducted very preliminary analyses of test-retest reliability $(n=11)$ at 2-3 weeks and 2-3 months and found no significant differences for any domains between any testing times $(p=0.143-$ 0.670 ), Cronbach's $\alpha$ levels ranging from 0.691 to 1.00 and interclass correlation coefficients ranging from 0.824 to 1.00 .

Parents completed the SensOR scoring each item as a " 1 " if behaviors or activities "bother" their child, or a " 0 " if the behaviors or activities are not bothersome. Items include such things as tags in clothing, cutting toenails or fingernails, fluorescent lights, slimy or lumpy foods, sirens or alarms, and going on amusement park rides. Subtest and total scores were added by the examiner. Domain scores on the SensOR that were greater than two standard deviations from normative mean in at least one category were considered atypical, and children with these atypical scores were grouped into the SOR category (ADHDs, TYPs). This method of classification had been used during our pilot work, and was useful in predicting groups that showed meaningful physiologic differences (Reynolds et al., 2009).

\section{Revised Children's Manifest Anxiety Scale}

The RCMAS is a 37-item self-report tool used to measure anxiety in children ages 6-19 years old (Reynolds and Richmond, 2005). It includes 28 items that measure traits related to anxiety, and nine

${ }^{1}$ http://www.psylab.com items that comprise a Lie Scale or social desirability score (e.g., I am always good). A total anxiety score and subscale scores (physiologic anxiety, worry/over-sensitivity, and social concern/concentration) are calculated. The RCMAS has been shown to have high internal consistency reliability and construct validity (Muris et al., 2002). High Lie Scale scores may reflect inaccurate self report, idealized sense of self, or inattention during the questionnaire process, providing a safeguard for child report. No children in the current study scored above 13 on the Lie Scale, and therefore, all RCMAS scores were considered valid for analysis. Higher scores on the RCMAS indicate greater levels of anxiety.

\section{Electrodermal response measures}

A measure of eccrine sweat gland activity, EDR reflect sympathetic nervous system responses to sensory stimuli. EDR were assessed during a 3-min baseline, throughout the SCP, and during a 3-min recovery. The general method of collecting EDR followed the procedures recommended by Fowles et al. (1981), and previously reported by Miller and colleagues (McIntosh et al., 1999b; Miller et al., 1999). Two 5-mm electrodes were applied to the thenar and hypothenar surface of the right hand, secured with double-sided sticky collars, then wrapped with Coban to ensure that the electrodes remained in place. These locations have previously been documented as adequate for recording purposes (Boucsein, 1992). The electrodes were attached to a PsyLab SC5 coupler, which digitizes skin conductance at the point where electrodes plug into the pre-amplifier, using a 24-bit A-D converter. A constant $0.5 \mathrm{~V}$ potential was applied across the electrode pair. A low-cut filter set to $0.2 \mathrm{~Hz}$ was used; signals greater than 0.2 are passed without distortion with respect to amplitude. The signals were sampled at $80 \mathrm{~Hz}$, then digitized and stored on a computer. For each subject, the electrodermal record was visually checked for movement artifact and questionable responses removed. PsyLab measurement software was used for wave detection; specific responses were taken as occurring between 0.8 and $3.999 \mathrm{~s}$ after the stimulus; responses before and after this window were considered non-specific responses (NSR). The amplitude of the peaks was measured from the point at which the skin conductance increases sharply (i.e., baseline) to the point at which the conductance begins to fall (i.e., peak). Only peaks greater than $0.05 \mu \Omega$ (Dawson et al., 1990) were considered valid.

A variety of measurement parameters of EDR have been used to assess tonic and phasic sympathetic activity and response to sensation, both of interest in the current study. In this study, tonic electrodermal activity, or skin conductance level (SCL) was averaged across a 3-min baseline (SCLbase) and during a 3-min recovery (SCLrec). Tonic activity was also reflected in the number of NSR recorded prior to beginning the SCP (NSRbase), and during a 3-min recovery following the SCP (NSRrec). The phasic, or reactivity, variables used in the current study included magnitude of response to the first stimulus of each domain (magOR) and mean response magnitude within each domain (e.g., magTactile). As is typical for studies evaluating magnitude of skin conductance responses, our magnitude data required logarithmic transformation before analysis (Dawson et al., 1990; Boucsein, 1992). 


\section{Salivary cortisol}

Subjects were instructed not to eat, drink, chew gum, or brush their teeth for $30 \mathrm{~min}$ before coming to the lab. Subjects rinsed their mouth thoroughly with cold water 5 min prior to beginning sample collection. In addition, parents were asked to withhold their child's ADHD medication (if applicable) for $24 \mathrm{~h}$ before testing to eliminate any potential neurochemical interactions and inter-subject variation.

Saliva was collected using plain (non-citric acid) cotton Salivettes $\left(\right.$ Sarstedt $t^{\circledast}$ ). Samples were maintained at room temperature until the child's session was complete, at which time they were labeled and frozen at $-20^{\circ} \mathrm{C}$ until analysis. This method of collection and storage has been recommended by Salimetrics LLC ${ }^{2}$.

A well defined diurnal rhythm of salivary cortisol has been shown in children over 1 year of age (Hiramatsu, 1981; Kiess et al., 1995). This rhythm is characterized by a steep rise in cortisol levels in the first 30-45 min after awakening followed by a steep decrease over the next $3 \mathrm{~h}$ (Edwards et al., 2001). In the 3-9 h after awakening cortisol levels have been shown to be most stabilized, slowly declining until evening hours (Edwards et al., 2001). Therefore all children were tested on non-school days and between the hours of $1 \mathrm{pm}$ and $4 \mathrm{pm}$ in attempt to limit variation due to normal diurnal patterns. The first baseline cortisol measure was taken after the child had completed the WASI, rinsed their mouth out with cold water, washed their hands, had their questions answered, and were comfortably seated in the mock space chair. A second baseline sample was taken after the accommodation period for electrode placement. A single baseline cortisol value (cort_bl) was obtained by averaging the two collected cortisol baseline samples. Changes in cortisol levels take approximately $5 \mathrm{~min}$ to register and approximately 15-20 min to peak in human saliva (Schmidt, 1997; Hiramatsu, 1981). The entire SCP takes approximately 15-20 min to complete. To capture cortisol responses to sensory challenge, seven saliva samples were collected post-SCP at $5 \mathrm{~min}$ intervals. For the purposes of this study, two post-challenge measures were considered: cortisol immediately post-challenge

${ }^{2} \mathrm{http}: / /$ www.salimetrics.com/cortisolkitinsert.htm
(cort_0P) and an average of cortisol levels at 25 and 30 min post challenge (cort_lastP).. In previous work we had determined that these later two cortisol measurements best reflected group differences (Reynolds et al., 2009).

Samples were analyzed in duplicate at the General Clinical Research Center (GCRC) at Virginia Commonwealth University using a high sensitivity salivary cortisol enzyme immunoassay kit (HS-Cortisol kit) produced by Salimetrics LLC. All samples remained frozen prior to assay and then were centrifuged at $1500 \times g$ at $3000 \mathrm{rpm} / \mathrm{min}$ for $15 \mathrm{~min}$.

\section{RESULTS \\ SenSOR}

All analyses reported were run with the full sample of 84 children. The actual $n$ within each analysis varies to some extent due to missing data. SenSOR scores were used to subdivide TYP and ADHD children into two subgroups each: TYP with SOR (TYPs) and without (TYP), ADHD with SOR (ADHDs) and without (ADHDt). Scores on the SenSOR reflect the number of item prompts that were "bothersome" to the child. Scores at or above 10 in tactile, 5 in auditory, 4 in taste, 2 in smell, 2 in visual, and 4 in movement were outside of $2 \mathrm{SD}$ and qualified the child as having SOR. SenSOR raw scores for included are presented in Table 1. TYPs and ADHDs groups demonstrated SOR. ANOVA results indicated significant differences between groups for all domains [Tactile: $F(3,79)=24.06$, $p<0.000$; Auditory: $F(3,79)=8.21, p<0.000$; Taste, $F(3,79)=8.38$, $p<0.000$; Smell: $F(3,79)=6.96, p<0.000$; Visual: $F(3,79)=11.33$, $p<0.000$; Movement: $F(3,79)=3.79, p<0.012]$. Significant results from post hoc comparisons using a Bonferroni analysis are noted in Table 1. Note that TYP and ADHDt did not differ from each, and TYPs and ADHDs did not differ from each other within any domain. Differences within other domains varied.

\section{DEMOGRAPHICS}

Analysis of variance indicated that groups did not differ on age, race, or ethnicity. The majority of children in all groups were white, non-hispanic. Although sex differences between groups were not significant $\left[\chi^{2}(3, n=83)=5.049, p=0.168\right)$, sex was not evenly distributed.

Table 1 | SensOR scores.

\begin{tabular}{|c|c|c|c|c|c|c|}
\hline SensOR domains ( $n$ items) & Tactile (28) & Auditory (20) & Taste (9) & Smell $(5)^{*}$ & Visual $(5)^{*}$ & Movement (9) \\
\hline $\operatorname{TYP}(n=36)$ & $2.86 \pm 2.87^{2,3}$ & $0.28 \pm 0.57^{3}$ & $1.19 \pm 1.21^{2,3}$ & $1.17 \pm 1.42^{2,3}$ & $0.14 \pm 0.35^{3}$ & $0.28 \pm 0.74^{3}$ \\
\hline TYPs $(n=9)$ & $8.22 \pm 3.83^{1,4}$ & $1.44 \pm 1.42$ & $3.33 \pm 1.80^{4}$ & $3.22 \pm 2.17^{1,4}$ & $1.22 \pm 1.09$ & $0.78 \pm 0.97$ \\
\hline ADHDt $(n=18)$ & $2.83 \pm 2.09^{2,3}$ & $0.39 \pm 0.61^{3}$ & $1.44 \pm 1.04^{2,3}$ & $1.06 \pm 1.00^{2,3}$ & $0.50 \pm 0.86^{3}$ & $0.28 \pm 0.57^{3}$ \\
\hline ADHDs $(n=21)$ & $10.09 \pm 4.87^{1,4}$ & $2.05 \pm 2.46^{1,4}$ & $3.24 \pm 2.64^{1,4}$ & $3.05 \pm 2.77^{1,4}$ & $1.90 \pm 1.95^{1,4}$ & $1.24 \pm 1.81^{1}$ \\
\hline
\end{tabular}

*Scores that reflect sensory over-responsivity.

Significant differences as follows:

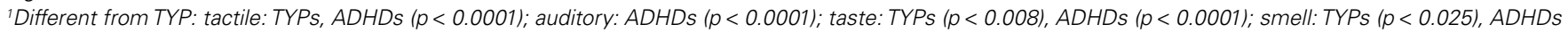
$(p<0.003)$; vision: $A D H D s(p<0.0001)$; movement: $A D H D s(p<0.014)$.

${ }^{2}$ Different from TYPs: tactile: TYP ( $\left.<<0.0001\right)$, ADHDt $(p<0.002)$; taste: TYP $(p<0.008)$, ADHDt $(p<0.052)$; smell: TYP ( $\left.<<0.025\right)$, ADHDt (p< 0.034).

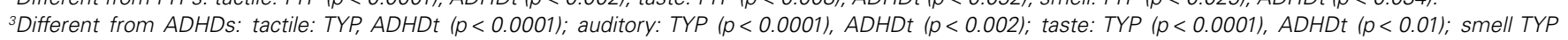
( $p<0.003)$, ADHDt $(p<0.008)$; vision: TYP $(p<0.0001)$, ADHDt $(p<0.001)$; movement: TYP $(p<0.014), A D H D t(p<0.051)$.

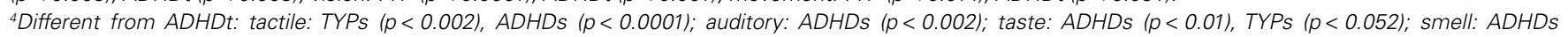
$(p<0.008)$, TYPs $(p<0.034)$; vision: $A D H D s(p<0.001)$; movement: $A D H D s(p<0.051)$. 
IQ was obtained for 84 children included in the final sample. All children fell within a typical IQ range, however, there were IQ differences between groups $[F(3,80), p=0.019)$. Bonferroni post hoc analysis identified this as a difference between TYPs and ADHSs subjects (Table 2).

\section{PHYSIOLOGIC VARIABLES}

Physiologic variables were chosen as a reflection of both tonic and phasic responsivity (Table 3). Tonic variables included baseline cortisol (cort_bl), natural log of SCL at baseline (SCLbase), and NSR at baseline (NSRbase). Phasic variables examined included natural log of orienting response magnitude (magOR), mean domain response magnitude (e.g., magTactile), natural log of SCL during recover (SCLrec) and NSR during recover (NSRrec). In addition, post-SCP cortisol measures were included as phasic or response variables (cort_0P, cort_lastP).

Multivariate analysis of variance tests were conducted in order to examine group differences for both tonic and phasic variables. Using baseline cortisol (cort_bl) and EDR variables (SCLbase and

Table 2 | Demographic information.

\begin{tabular}{lcccr}
\hline & $\begin{array}{l}\text { TYP } \\
(\boldsymbol{n}=\mathbf{3 6})\end{array}$ & $\begin{array}{l}\text { TYPs } \\
(\boldsymbol{n}=\mathbf{9})\end{array}$ & $\begin{array}{l}\text { ADHDt } \\
(\boldsymbol{n}=\mathbf{1 8})\end{array}$ & $\begin{array}{r}\text { ADHDs } \\
(\boldsymbol{n}=\mathbf{2 1})\end{array}$ \\
\hline Age & 102.056 & 105.55 & 101.39 & 101.62 \\
& \pm 20.06 & \pm 25.34 & \pm 24.00 & \pm 20.57 \\
\multicolumn{2}{l}{ SEX DISTRIBUTION } & & & \\
Girls $(n)$ & 19 & 5 & 5 & 6 \\
Boys $(n)$ & 17 & 4 & 13 & 15 \\
IO & 112.12 & 118.63 & 111.18 & 100.90 \\
& \pm 15.04 & $\pm 9.86^{*}$ & \pm 17.54 & $\pm 14.70^{*}$ \\
\hline
\end{tabular}

${ }^{*}$ TYPs and ADHDs, $p=0.039$.

Table 3 | Physiologic and neuroendocrine variables and their abbreviations.

\begin{tabular}{ll}
\hline & Abbreviation \\
\hline TONIC VARIABLES & \\
Cortisol at Baseline & cort_bl \\
Log skin conductance level at baseline & SCLbase \\
Non-specific electrodermal responses & NSRbase \\
at baseline & \\
PHASIC VARIABLES & \\
Cortisol 0 min post-challenge & cort_OP \\
Cortisol at 25/30 min post-challenge & cort_lastP \\
Log magnitude of electrodermal response & magOR \\
to first stimulus in each domain & \\
(orienting response) & \\
Log mean magnitude of electrodermal & \\
response in each sensory domain & magTone, magVisual, \\
& magSiren, magSmell, \\
Log skin conductance level during recovery & magTactile, magMove \\
Non-specific electrodermal & SCLrec \\
responses during recovery & NSRrec
\end{tabular}

NSRbase) no between group differences were found. In the analysis of phasic measures cortisol and EDR were examined separately because they follow a different time course. Examination of phasic EDR magnitude of response within each domain indicated no between group differences; no significant differences were found for cortisol levels at baseline or following SCP (Figure 1). Using SCLrec and NSRrec as reflections of recovery from the sensory challenge, group differences were identified [Wilks' Lambda $F(6,130)=2.304$, $p<0.038]$. Post hoc Bonferroni analyses indicated differences between TYP and ADHDs groups $(p<0.039)$ for NSRrec.

\section{ANXIETY}

The three subscales of the RCMAS were examined to determine group differences (RPA: physiologic anxiety; RWO: worry/over-sensitivity; RSCC: social concern/concentration). Multivariate analysis of variance indicated a significant group difference [Wilks' Lambda $F(9,187.548)=7.073, p<0.000)$. Post hoc analyses are presented in Table 3. The subscale anxiety scores for each group are plotted in Figure 2. Post hoc analysis using a Bonferroni procedure indicated that children with ADHDs differed from TYP children on all scales $(p<0.000)$, and from children with ADHDt on the RPA $(p<0.000)$, marginally on the RWO subscale $(p<0.054)$, but not RSCC $(p=0.313)$. TYP and ADHDt children were significantly different only for the RSCC scale (RPA, $p<0.657$; RWO, $p<0.194$; RSCC, $p<0.001$ ). TYPs children differed from ADHDs on the RPA $(p<0.031)$, the RWO $(p<0.009)$, and the RSCC $(p<0.036)$ subscales.

Anxiety is considered clinically relevant when the total score on the RCMAS (RTA = total anxiety score) exceeds 19 . Overall anxiety mean scores are presented in Table 4. While mean scores indicate no clinically relevant anxiety, one ADHDt child attained a score above 19, and eight ADHDs children attained a score above 19. No TYP or TYPs children attained this score.

\section{GROUP CLASSIFICATION}

A stepwise linear discriminant analysis was conducted to determine if physiologic (EDR), neuroendocrine (cortisol) and anxiety variables could meaningfully predict group membership for the sample. We

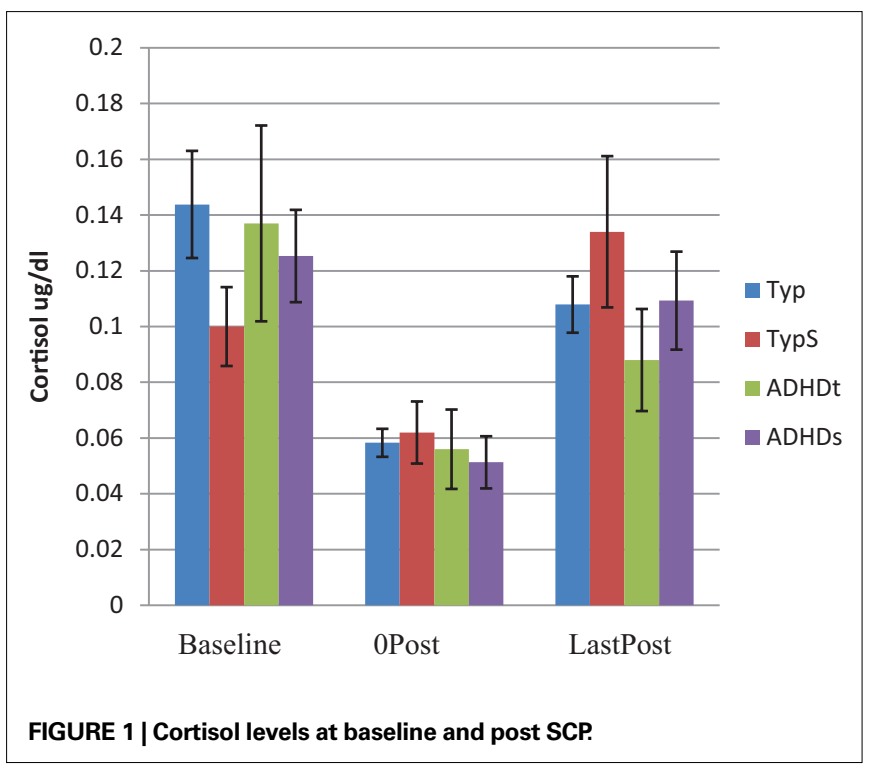


chose to use tonic and phasic variables for both EDR and cortisol, as well as anxiety subscale scores to examine all potential contributors to group membership. The stepwise analysis was conducted using three methods; forward selection, backward elimination and stepwise (forward and backward) options. The forward selection and stepwise options selected the same variables to form the linear discriminating function while the backward elimination procedure selected a different set of variables. The variables entered into the analysis for consideration for the discriminant function included those reflecting anxiety [physiologic anxiety (RPA), worry/over-sensitivity (RWO) and social concern/concentration (RSCC)], natural log of EDR at baseline [log of baseline SCL (SCLbase and SCLrec) and number of NSR at baseline and recovery (NSRbase, NSRrec)], baseline cortisol (cort_bl), natural log of phasic EDR [overall orienting response magnitude (magOR), magnitude of natural log values for response to tone, visual, siren, smell, touch, movement], and phasic cortisol [cortisol immediately post challenge (cort_0P), and cortisol at 25/30 min post-challenge (cort_lastP)]. Children with missing data were excluded from the linear discriminant analysis, resulting in an $n=59$. The linear discriminant functions derived from the forward selection and stepwise procedures are shown in Table 5. As can be seen here, only RPA and RSCC contributed to the function. Based on these values, group classification was examined on all children for whom these values were available $(n=84)$. Group classification based upon these discriminant functions is shown in Table 6.

The discriminant function allowed us to correctly classify $58.3 \%$ (21/36) of TYP children, $61.9 \%$ (13/21) of ADHDs children, $44.4 \%$ (8/18) of the ADHDt children and $44.4 \%$ (4/9) of the TYPs children. On average this discriminating function correctly classified $52.3 \%$ of the children in the sample.

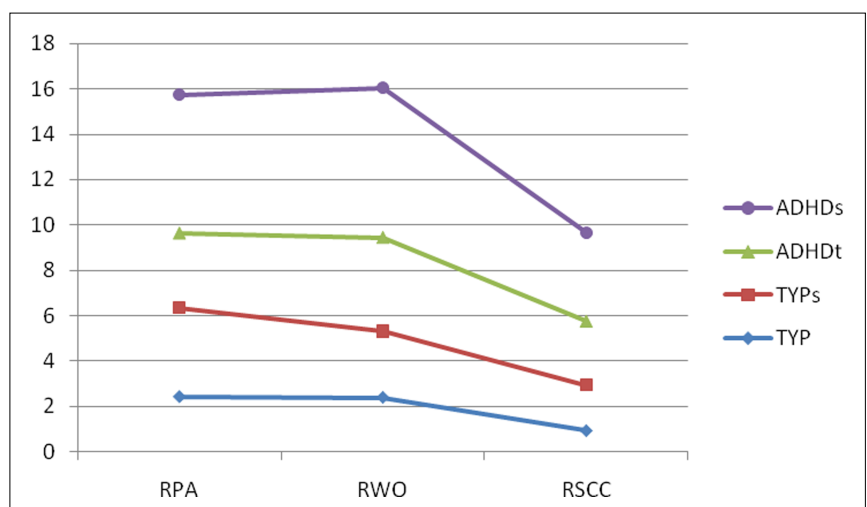

FIGURE 2 |Anxiety scores. RPA, physiologic anxiety; RWO, worry/oversensitivity; RSCC, social concern/concentration; TYP, typical children; TYPs, typical children with sensory over-responsiveness; ADHDt, ADHD without sensory over-responsiveness; ADHDs, ADHD with sensory overresponsiveness.

Table 4 | Mean total anxiety scores.

\begin{tabular}{lrr}
\hline & \multicolumn{1}{c}{$\boldsymbol{n}$} & Mean \pm SD \\
\hline TYP & 35 & $5.83 \pm 3.97$ \\
TYPs & 9 & $8.78 \pm 3.35$ \\
ADHDt & 18 & $10.39 \pm 5.36$ \\
ADHDs & 21 & $16.67 \pm 6.81$
\end{tabular}

The linear discriminant function derived from the backward elimination procedure is shown in Table 7. As can be seen here, RPA, RSCC, NSRbase, SCLrec, magTone, magSiren and magOlf all contributed to the function. Based on these values, group classification was examined on all children for whom these values were available $(n=71)$. Group classification based upon these discriminant functions is shown in Table 8.

This discriminant function allowed us to correctly classify $74.2 \%$ (23/31) of TYP children, $72.2 \%$ (13/18) of ADHDs children, $50.0 \%$ (7/14) of the ADHDt children and 62.5\% (5/8) of the TYPs children. On average this discriminating function correctly classified $64.7 \%$ of the children in the sample.

When a linear discriminant analysis was conducted with all of the physiologic, neuroendocrine and anxiety variables the linear discriminant functions for the 59 children with complete data can be seen in Table 9. Group classification based upon these discriminant functions is shown in Table $\mathbf{1 0 .}$

Table 5 | Linear discriminant function derived from forward selection and stepwise procedure for groups.

\begin{tabular}{lrrrr}
\hline Variable & TYP & ADHDs & ADHDt & TYPs \\
\hline Constant & -0.59 & -6.79 & -2.14 & -1.96 \\
RPA & 0.56 & 1.67 & 0.75 & 0.91 \\
RSCC & 0.08 & 0.73 & 0.67 & 0.37 \\
\hline
\end{tabular}

RPA, physiologic anxiety; RSCC, social concern/concentration ( $n=59)$.

Table 6 | Classification results $(n=84)$.

\begin{tabular}{lccccc}
\hline GROUP & $\begin{array}{l}\text { Classified } \\
\text { TYP }\end{array}$ & $\begin{array}{l}\text { Classified } \\
\text { ADHDs }\end{array}$ & $\begin{array}{l}\text { Classified } \\
\text { ADHDt }\end{array}$ & $\begin{array}{l}\text { Classified } \\
\text { TYPs }\end{array}$ & Total \\
\hline TYPICAL & 21 & 3 & 2 & 10 & 36 \\
ADHDs & 1 & 13 & 4 & 3 & 21 \\
ADHDt & 4 & 3 & 8 & 3 & 18 \\
TYPs & 1 & 0 & 4 & 4 & 9 \\
Total & 27 & 19 & 18 & 20 & 84
\end{tabular}

Table 7 | Linear discriminant function derived from backward elimination for groups.

\begin{tabular}{lrrrr}
\hline Variable & TYP & ADHDs & ADHDt & TYPs \\
\hline Constant & -6.84 & -16.89 & -10.35 & -7.93 \\
RPA & 0.70 & 2.19 & 1.11 & 1.39 \\
RSCC & 0.58 & 1.31 & 1.23 & 0.71 \\
NSRbase & -0.16 & 0.07 & -0.06 & 0.25 \\
SCLrec & 0.79 & 0.97 & 0.95 & 0.55 \\
magTone & -4.00 & -3.65 & -4.37 & -0.58 \\
magSiren & -2.95 & -5.74 & -4.15 & -5.48 \\
magOlf & -3.08 & -0.15 & -1.26 & 0.41 \\
\hline
\end{tabular}

RPA, physiologic anxiety; RSCC, social concern/concentration; NSRbase, non-specific electrodermal responses at baseline; SCLrec, natural log of skin conductance level at baseline; magTone, magSiren, magOlf, natural log mean magnitude of electrodermal response for tone, siren, olfactory, respectively $(n=59)$. 
The discriminant function allowed us to correctly classify $79.2 \%$ (19/24) of TYP children, 88.2\% (15/17) of ADHDs children, 91.7\% (11/12) of the ADHDt children and $83.3 \%$ (5/6) of the TYPs children. On average this discriminating function correctly classified $85.6 \%$ of the children in the sample. So, while the most predictive variables of group membership, based upon the stepwise procedures, are the anxiety variables RPA and RSCC (forward and stepwise analysis), or the anxiety variables RPA and RSCC along with EDR variables (NSRbase, SCLrec, magTone, magSiren, and magOlf) (backward analysis), the overall correct classification rate

Table 8 | Classification results $(n=71)$.

\begin{tabular}{lccllr}
\hline GROUP & $\begin{array}{l}\text { Classified } \\
\text { TYP }\end{array}$ & $\begin{array}{l}\text { Classified } \\
\text { ADHDs }\end{array}$ & $\begin{array}{l}\text { Classified } \\
\text { ADHDt }\end{array}$ & $\begin{array}{l}\text { Classified } \\
\text { TYPs }\end{array}$ & Total \\
\hline TYPICAL & 23 & 0 & 3 & 5 & 31 \\
ADHDs & 0 & 13 & 4 & 1 & 18 \\
ADHDt & 4 & 2 & 7 & 1 & 14 \\
TYPs & 1 & 1 & 1 & 5 & 8 \\
Total & 28 & 16 & 15 & 12 & 71
\end{tabular}

Table 9 | Linear discriminant function for groups based upon physiologic, neuroendocrine and anxiety variables $(n=59)$.

\begin{tabular}{lrrrr}
\hline Variable & \multicolumn{1}{c}{ TYP } & ADHDs & ADHDt & \multicolumn{1}{c}{ TYPs } \\
\hline Constant & -15.10 & -26.10 & -16.60 & -13.92 \\
RPA & 0.69 & 2.23 & 1.17 & 1.40 \\
RWO & 0.21 & 0.42 & 0.15 & 0.20 \\
RSCC & 0.18 & 0.77 & 0.99 & 0.38 \\
NSRbase & -0.58 & -0.40 & -0.39 & -0.18 \\
NSRrec & 0.31 & 0.45 & 0.16 & 0.28 \\
SCLbase & 2.57 & 2.76 & 2.14 & 4.67 \\
SCLrec & 0.93 & 1.00 & 1.08 & 0.37 \\
cort_bl & 48.38 & 53.59 & 48.02 & 30.17 \\
Cort_OP & 75.15 & 59.72 & 72.68 & 61.78 \\
cort_lastP & 1.89 & 5.96 & -9.14 & 5.14 \\
magOR & -2.41 & -2.25 & -0.54 & -2.80 \\
magTone & -5.78 & -5.84 & -5.97 & -1.60 \\
magVis & -2.19 & -4.42 & -1.90 & -2.20 \\
magSiren & -4.46 & -7.43 & -5.28 & -6.76 \\
magOIf & -3.20 & 0.95 & -1.03 & 1.55 \\
magTact & 1.96 & 0.41 & -0.10 & 1.53 \\
magMove & -0.22 & 2.55 & -0.58 & 0.96
\end{tabular}

Table 10 | Classification results $(n=59)$.

\begin{tabular}{llllll}
\hline GROUP & $\begin{array}{l}\text { Classified } \\
\text { TYP }\end{array}$ & $\begin{array}{l}\text { Classified } \\
\text { ADHDs }\end{array}$ & $\begin{array}{l}\text { Classified } \\
\text { ADHDt }\end{array}$ & $\begin{array}{l}\text { Classified } \\
\text { TYPs }\end{array}$ & Total \\
\hline TYPICAL & 19 & 0 & 3 & 2 & 24 \\
ADHDs & 0 & 15 & 2 & 0 & 17 \\
ADHDt & 1 & 0 & 11 & 0 & 12 \\
TYPs & 1 & 0 & 0 & 5 & 6 \\
Total & 21 & 15 & 16 & 7 & 59
\end{tabular}

with variables from all three domains $(85.6 \%)$ is better than the overall classification rate with only the variables from the stepwise procedures ( 52.3 or $64.7 \%$ ).

It should be noted that since we are classifying the same data that was used to derive the discriminating function, these rates are biased. One way to reduce this bias is to classify each subject using a discriminant function computed from all the other children (cross validation). When we cross validated our data we were able to correctly classify $62.5 \%(15 / 24)$ of the TYP children, $58.8 \%$ of the ADHDs (10/17) children, $25.0 \%$ of the ADHDt (3/12) children and $33.3 \%(2 / 6)$ of the TYPs children; on average $44.9 \%$ of the children (Table 11).

\section{DISCUSSION}

$\mathrm{ADHD}$ has long been viewed as a heterogeneous diagnostic group, and many investigators have examined the current subgroupings (i.e., inattentive, hyperactive, combined type) that define these children (cf. Barkley, 2001; Milich et al., 2001; Adams et al., 2008). Other researchers have considered how comorbid conditions, which consistently present in children with ADHD, affect presentation of symptoms and response to treatment. Pliszka (2003) suggests that comorbidity with conditions such as CD, ODD, and anxiety disorder appear to characterize distinct subtypes of ADHD. In the current study we examined a somewhat different aspect of $\mathrm{ADHD}$, that related to the processing of sensation. We had previously documented the existence of SOR in a small group of children with $\mathrm{ADHD}$, and suggested that it might serve as a moderating variable for ADHD subgroups (Reynolds et al., 2009). Here we substantiate this finding, linking SOR to anxiety, and identify predictor variables for SOR.

We successfully classified children with ADHD into two groups: with and without SOR. While this is not a new finding (Reynolds et al., 2009), it is robust. In the current sample $46 \%$ of the children with ADHD also had SOR. While this is a large percentage, it is somewhat lower than that reported by Parush et al. (2007) for tactile over-responsivity in boys with ADHD. Sensory processing disorders have not routinely been considered in conjunction with ADHD, but their identification is becoming more common. Interestingly, we were also able to classify our typical children as those with and without SOR; in the current sample $20 \%$ of our otherwise typical children showed SOR. That there are children otherwise considered to be "typical” with SOR has been previously indicated (McIntosh et al., 1999b; Reynolds and Lane, 2007), but disorders of sensory modulation are not currently recognized as an ICD-10 or DSM-IV diagnosis (Cheng and Boggett-Carsjens, 2005). Our findings suggest that this should be investigated in greater depth.

Table 11 | Cross-validated classification summary using the linear discriminant function.

\begin{tabular}{lccllr}
\hline GROUPSR & $\begin{array}{l}\text { Classified } \\
\text { TYP }\end{array}$ & $\begin{array}{l}\text { Classified } \\
\text { ADHDs }\end{array}$ & $\begin{array}{l}\text { Classified } \\
\text { ADHDt }\end{array}$ & $\begin{array}{l}\text { Classified } \\
\text { TYPs }\end{array}$ & Total \\
\hline TYPICAL & 15 & 1 & 6 & 2 & 24 \\
ADHDs & 1 & 10 & 3 & 3 & 17 \\
ADHDt & 4 & 2 & 3 & 3 & 12 \\
TYPs & 2 & 1 & 1 & 2 & 6 \\
Total & 22 & 14 & 13 & 10 & 59
\end{tabular}


SOR could be described as a "tonic" or steady state characteristic. It is a response to every day sensation that is of greater intensity or longer duration than would be expected given the nature of the stimulus (Miller et al., 2007). Some children with SOR can be characterized as passive in their response to sensory input (Dunn, 1999); they may be withdrawn in an effort to avoid the sensation in the environment which they find bothersome (Miller et al., 2007). Other children with SOR seek to actively escape sensory input they find unpleasant, becoming restless and potentially aggressive in their need to avoid sensation. Both groups of children may be hypervigilant within their environment. These observable behaviors of SOR parallel those identified in children with ADHD, especially the hyperactive-impulsive type, which is characterized by behaviors such as fidgeting with hands or feet, squirming, and difficulty waiting turns (American Psychiatric Association, 2000). Our data indicate that SOR and ADHD can be linked, but importantly that they are seen separately. Sonuga-Barke (2005) had suggested that the hyperactive-impulsive symptoms of ADHD were linked to meso-limbic dopamine system differences, impacting motivation linked behavioral control. Drawing on this Nigg (2006) described these behaviors as related to reactive control, linked to "bottom-up" processes that are stimulus driven, and potentially linked to striatal or limbic activation. The link to reactive control may also relate to SOR. Some children with SOR respond to sensory stimuli they find bothersome by striking out or moving to escape the offending input; they perceive the input as potentially dangerous and react accordingly. Specific CNS loci related to SOR have not been identified.

Not surprisingly, anxiety was identified in children with ADHD. This link has been documented by many earlier investigators (e.g., Pliszka, 1998, 2000; Jensen et al., 2001). In the current study, children with ADHDs differed from typical children on all anxiety subscales, indicating a very broad link between anxiety and SOR in this subgroup. Of interest to the current study, Jensen et al. (1997) suggested that there may be a specific subgroup of children with ADHD and anxiety that sets them apart from children with ADHD but not anxiety; our findings lend support to this suggestion as we document that children with ADHDs are different from both typical children, and from children with ADHD but no SOR. We have repeated earlier, preliminary, findings that children with ADHD and SOR are more likely to show total anxiety that is clinically relevant than are children with ADHD but no SOR (Reynolds and Lane, 2009). In this study, as well as previously, children with ADHDs showed higher anxiety on all subscales of the RSMAS. Hofmann and Bitran (2007) had also documented the co-existence of sensory-processing sensitivity and anxiety disorder. These investigators had specifically identified social anxiety disorder in adults as linked to what we would term SOR. Extending this to children, we found that children with ADHD and SOR showed higher anxiety on the social concern/concentration subscale when compared to typical children, but interestingly not when compared to children with ADHD and no SOR. Thus, ADHD appears to be linked with elevated anxiety on social concern/concentration, regardless of sensory over-responsivity. Thus, some aspects of anxiety may characterize ADHD generally (anxiety related to social concern/concentration), while other aspects of anxiety may be more specifically tied to SOR (physiologic anxiety and possibly worry/over-sensitivity).
Diagnostically, it is likely that ADHD, SOR, and anxiety disorder exist independently, yet overlap consistently in ways that influence the behavioral presentation of the child (Figure 3).

In considering children with ADHD and anxiety, Levy (2004) postulated a deficit in prefrontal cortex/hippocampal synaptic gating, allowing access to amygdalar fear reactions. Given that sensory input influences amygdalar function, the combination of SOR, anxiety, and ADHD may have a similar linkage. Levy linked the gating deficit to noradrenergic and serotonergic amygdala responses. Support for Levy's finding comes from studies noting that children with ADHD and anxiety tend to be poor medication responders, indicating that the link with catecholamine dysfunction may not be clear in this group (Levy, 2004). Children with ADHD + anxiety have been noted to respond to a blend of medication and behavioral intervention. The finding here that some children with ADHD + anxiety also had SOR suggests that there may be more to this group of children than has been previously identified. Intervention for SOR takes the form of behavioral treatment for the child, along with parent education and environmental adaptation (Parham et al., 2007). This approach could easily be integrated with current behavioral interventions for ADHD, potentially providing a more potent scope of treatment.

Although anxiety in the typical group as a whole did not reach a clinically significant level, anxiety subscale scores differed between typical children with and without SOR. This finding is certainly not diagnostic, but rather it provides information on SOR in otherwise typical children, and indicates that the relationship between SOR and anxiety is not dependent on a diagnosis of ADHD. Based on the work of Gray (1975), Royeen and Lane (1992) had suggested a link between sensory defensiveness (over-responsivity) and the amygdalar fear response. The somewhat elevated anxiety seen in the TYPs children in this study lend some support for this. The presence of SOR in the absence of other diagnoses has been demonstrated by other investigators (Kinnealey and Fuiek, 1999; Mangeot et al., 2001; Reynolds and Lane, 2007).

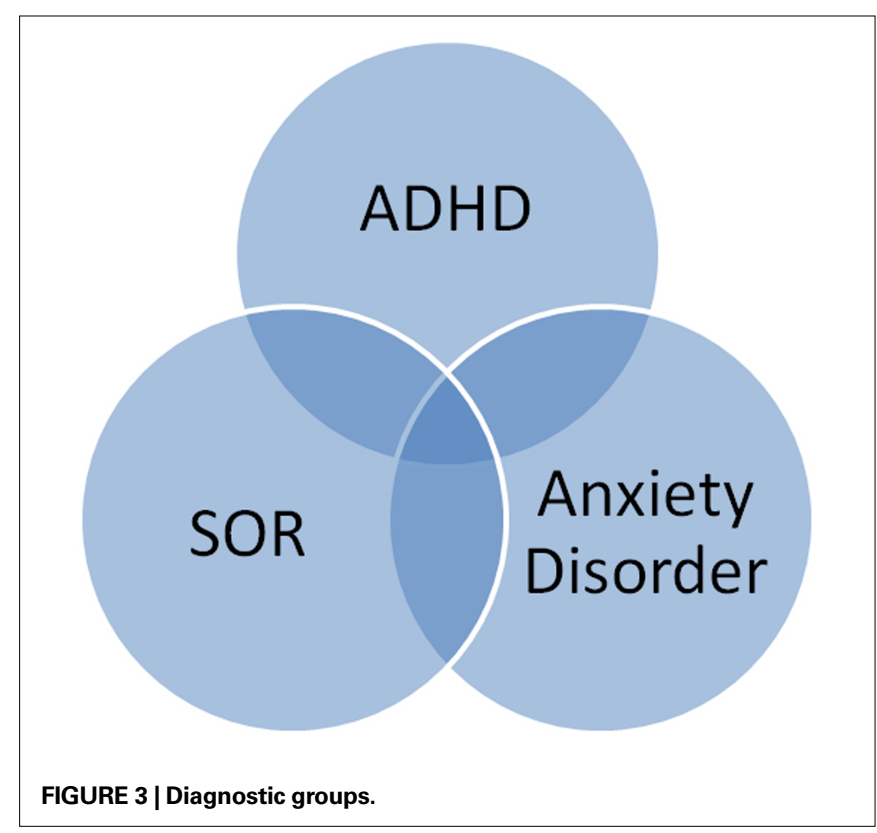


We set out to determine if children with ADHDs could be differentiated from children with ADHD but no SOR (ADHDt) and typical (TYP) children based on neuroendocrine and electrodermal markers. Examined individually, one differentiating marker was identified: EDR non-specific response during recovery. Children with ADHDs differed from typical children in their ability to recover from the sensory challenge. NSR have been linked physiologically to increased tonic arousal (Venables and Christie, 1980). The ADHDs children showed over twice as many non-specific EDA spikes post-challenge, indicative of high arousal levels during recovery from sensory challenge. This ongoing behavior may be another reflection of reactive control in this group of children. Ognibene (2002) had suggested that children with ADHD could be distinguished based on EDR to sensory challenge, although he had failed to document differences. Mangeot et al. (2001) had documented marginally significant $(p<0.056)$ differences between children with and without ADHD using a similar sensory challenge. However, in both studies differences were found or expected during the sensory challenge, in response magnitude, habituation, or orienting. It is possible that the sensory challenge used in our study as well as these was insufficient to elicit a consistent sympathetic nervous system response. Examination of the paradigm is warranted.

Other investigators have examined EDR in children with ADHD, although not linked to sensory over-responsiveness. EDR differences have been documented in children with ADHD with comorbid diagnoses of ODD and CD, finding in both cases that attenuated EDR responses are seen with these comorbidities (McBurnett and Lahey, 1994; Crowell et al., 2006). Our investigations differed in that we excluded children with ODD or CD comorbidity, and in our experimental conditions, making direct comparisons less meaningful.

Changes in neuroendocrine stress responses alone, reflected in cortisol, did not differentiate statistically between groups in the current study. However, patterns of response paralleled what we had shown earlier with a smaller sample; cortisol response to SCP varies between groups with and without SOR. Due to the time that it takes for cortisol to peak in saliva $(-20 \mathrm{~min})$, the post challenge cortisol measure taken at 0 -min post challenge (cort_0P) is reflective of the children's hormonal stress level in the early minutes of the SCP. Interestingly, in the current study all groups had nearly equivalent cortisol levels at this time point. However, at 25/30 min post-challenge (cort_lastP) cortisol levels were elevated, although with considerable more variability, in the TYPs and ADHDs groups to a greater extent than in either of the other non-SOR groups. This cortisol response pattern in our children with ADHDs had been identified in our earlier work, and stands in contrast to other studies which found that children with ADHD and externalizing disorders tend to show blunted cortisol levels in response to a challenge or stressor (King et al., 1998; Hong et al., 2003; van West et al., 2009). The children in this study with ADHDt did show a more attenuated cortisol response, suggesting that SOR may influence the cortisol response in children with ADHD.

A primary goal of this investigation was to examine the relationship between behavioral measures of sensory processing, and anxiety, with physiologic measures of autonomic nervous system function; links that have been explored by other investigators although not in the context of sensory processing (Bauer et al., 2002). Using EDR and cortisol variables coupled with anxiety measures allowed us to classify children in this study better than when anxiety measures were used alone, suggesting that a multisystem analysis is best for characterizing these complex problems. While group differences were not statistically significant, children with ADHDs had higher cortisol levels than did other groups, at measurement points other than baseline; EDR responses varied during the sensory challenge, but were significantly higher in the ADHDs group during recovery. EDR is a reflection of activity in the sympatho-adrenal-medullary (SAM) system and cortisol is a reflection of the HPA system. While both systems are involved in the stress response, they are activated differently depending on context, and the individual's perception of context. Both systems contributed to the model and added to our ability to correctly classify children. However, it is important to note that the model used here continues to need refinement. When we cross validated our data, our ability to predict was diminished to only slightly better than chance. While we still correctly classified $\sim 63 \%$ of the TYP children and $\sim 59 \%$ of children with ADHDs. Prediction of membership in ADHDt and TYPs groups was below chance. Clearly other factors are involved in characterizing these groups that remain to be defined. Other investigators have indicated that measurement of parasympathetic functions may better differentiate children with ADHD (Schaaf et al., 2003; Crowell et al., 2006). We did not analyze parasympathetic parameters in this study.

In conclusion, the finding here of a group of children with ADHD that is set apart from both typical children and other children with ADHD by a combination of SOR and clinically relevant anxiety provides new insight into this very different group of children with ADHD that warrants consideration. While we do not suggest that the presence of SOR is central to the diagnosis of ADHD, the presence of SOR symptoms with or without anxiety may have different CNS reflections, and important implications for prescribing and evaluating treatment. Physiologic differences between groups will need to be more deeply investigated, and this will add to our understanding of etiology and treatment. Just as children with ADHD and comorbid anxiety respond better to behavioral treatment than children with ADHD alone (Pliszka, 2003); children with ADHD, anxiety, and SOR may also show more improvements in function and behavior when treatments target their specific sensory modulation deficits along with problems associated with ADHD and anxiety.

\section{LIMITATIONS}

This study utilized a relatively small convenience sample of children with ADHD. Because of our interest in the interaction among variables, the sample size was small relative to the variables examined. Many children were self-referred by parents interested in knowing more about their child's sensory responses and thus the percentage of children with both ADHD and SOR may be higher than found in the general population. In addition, controlling for secondary 
diagnoses in the ADHD group depended on parental report; it is possible that these children may have co-morbid conditions that were not diagnosed.

The SCP itself may present stimuli that are not sufficiently stressful to consistently elicit stress and sympathetic responses from children. Stimuli in this protocol were chosen because they mimic daily sensation; the goal was to provide input that challenged the sensory processing ability of the children, but did not elicit undue stress. The protocol may need to be further explored.

\section{CONCLUSIONS}

This study suggests that ADHD should be considered in conjunction with anxiety and sensory responsivity; both may be related to bottom-up processing differences, and deficits in prefrontal cortex/

\section{REFERENCES}

Adams, Z. W., Derefinko, K. J., Milich, R., and Fillmore, M. T. (2008). Inhibitory functioning across adhd subtypes: recent findings, clinical implications, and future directions. Dev. Disabil. Res. Rev. 14, 268-275.

American Psychiatric Association. (2000). Diagnostic and Statistical Manual of Mental Disorders, 4th Edn (Text Revision). Washington, DC: American Psychiatric Association.

Aron, E. N., and Aron, A. (1997). Sensory processing sensitivity and its relation to introversion and emotionality. $J$. Pers. Soc. Psychol. 73, 345-368.

Ayres, A. J. (1972). Sensory Integration and Learning Disorders. Los Angeles: Western Psychological Services.

Barkley R.A. (2001). The inattentive type of ADHD as a distinct disorder: what remains to be done. Clin. Psychol. Sci. Pract. 8, 489-493.

Bauer, A. M., Quas, J. A., and Boyce, W. T. (2002). Associations between physiological reactivity and children. J. Dev. Behav. Pediatr. 23, 102-113.

Boucsein, W. (1992). Electrodermal Activity. New York: Plenum Press.

Brown, C. E., and Dunn, W. (2002). Adolescent/Adult Sensory Profile: Users Manual. San Antonio, TX: The Psychological Corporation.

Cheng, M., and Boggett-Carsjens, J. (2005). Consider sensory processing disorders in the explosive child: Case report and review. Can. Child Adolesc. Psychiatr. Rev. 14, 44-48.

Crowell, S. E., Beauchaine, T. P., GatzkeKopp, L., Sylvers, P., Mead, H., and Chipman-Chacon, J. (2006). Autonomic correlates of attentiondeficit/hyperactivity disorder and oppositional defiant disorder in preschool children. J. Abnorm. Psychol. 115, 174-178.

Dawson, M. E., Schell, A. M., and Filion, D. L. (1990). "The electrodermal system," in Principles of Psychophysiology: Physical, Social, and Inferential Elements, eds J. T. Cacioppo and L.G. Tassinary (New York: Cambridge University Press), 294-324.

Dunn, W. (1999). The Sensory Profile: Users manual. San Antonio, TX: The Psychological Corporation.

Edwards, S., Clow, A., Evans, P., and Hucklebridge, F. (2001). Exploration of the awakening cortisol response in relation to diurnal cortisol secretory activity. Life Sci. 68, 2093-2103.

Fowles, D. C., Christie, M. J., Edelberg, R., Grings, W. W., Lykken, D. T., and Venables, P. H. (1981). Publication recommendations for electrodermal measurements. Psychophys. Res. 18, 232-239.

Gere, D. R., Capps, S. C., Mitchell, D. W., and Grubbs, E. (2009). Sensory sensitivities of gifted children. Am. J. Occup. Ther. 63, 288-295.

Gray, J. A. (1975). Elements of a Twoprocess Theory of Learning. London: Academic Press.

Hanrahan, K., McCarthy, A. M., Kleiber, C., Lutgendorf, S., and Tsalikian, E. (2006). Strategies for salivary cortisol collection and analysis in research with children. Appl. Nurs. Res. 19, 95-101.

Hiramatsu, R. (1981). Direct assay of cortisol in human saliva by solid phase radioimmunoassay and its clinical applications. Clin. Chim. Acta 117, 239-249.

Hofmann, S. G., and Bitran, S. (2007). Sensory-processing sensitivity in social anxiety disorder: relationship to harm avoidance and diagnostic subtypes. J. Anxiety Disord. 21, 944-954.

Hong, H. J., Shin, D. W., Lee, E. H., Oh, Y. H., and Noh, K. S. (2003). Hypothalamic-pituitary-adrenal reactivity in boys with attention deficit hyperactivity disorder. Yonsei Med. J. 44, 608-614.

Jensen, P. S., Hinshaw, S. P., Kraemer, H. C., Lenora, N., Newcorn, J.H., Abikoff, H. B., March, J. S., Arnold, L. E., Cantwell, D. P., Conners, C. K., Elliott,

hippocampal synaptic gating. A better understanding, both behaviorally and physiologically may result in more effective treatment. Further investigation is needed.

\section{ACKNOWLEDGMENTS}

We extend thanks to the families and children who have made this work possible. We would like to thank members of the Sensory Integration Research Collaborative for their thoughts and feedback throughout the research process. This research was supported by the National Institutes of Health General Clinical Research Center (Grant M01 RR00065, NCRR, NIH), the American Occupational Therapy Foundation, and the Virginia Commonwealth University A.D. Williams research development fund.

G. R., Greenhill, L. L., Hechtman, L. Hoza, B., Pelham, W. E., Severe, J. B., Swanson, J. M., Wells, K. C., Wigal, T., and Vitiello, B. (2001). ADHD comorbidity findings from the MTA study: comparing comorbid subgroups. $J$. Am. Acad. Child Adolesc. Psychiatry 40, 147-158.

Jensen, P. S., Martin, D., and Cantwell, D. P. (1997). Comorbidity in ADHD: implications for research, practice, and DSM-V. J. Am. Acad. Child Adolesc. Psychiatry 36, 1065-1079.

Johnson, F. N. (1975). Depression: some proposals for future research. Dis. Nerv. Syst. 36, 228-232.

Kariyawasam, S. H., Zaw, F., and Handley, S. L. (2002). Reduced salivary cortisol in children with comorbid attention deficit hyperactivity disorder and oppositional defiant disorder. Neuroendocrinol. Lett. 23, 45-48.

Kiess, W., Meidert, A., Dressendörfer, R. A., Schriever, K., Kessler, U., König, A., Schwarz, H. P., and Strasburger, C. J. (1995). Salivary cortisol levels throughout childhood and adolescence: relation with age, pubertal stage, and weight. Pediatr. Res. 37, 502-506.

King, J. A, Barkley, R. A., and Barrett, S. (1998).Attention-deficit hyperactivity disorder and the stress response. Biol. Psychiatry 44, 72-74.

Kinnealey, M., and Fuiek, M. (1999). The relationship between sensory defensiveness, anxiety, depression, and perception of pain in adults. Occup. Ther. Int. 6, 195-206.

Kinnealey, M., Oliver, B., and Wilbarger, P. (1995). A phenomenological study of sensory defensiveness in adults. Am. J. Occup. Ther. 49, 444-451.

Levy, F. (2004). Synaptic gating and ADHD: a biological theory of comorbidity of ADHD and anxiety. Neuropsychopharmacology 29, 1589-1596.

Mangeot, S. D., Miller, L. J., McIntosh, D. N., McGrath-Clarke, J., Simon,
J., Hagerman, R. J., and Goldson, E. (2001). Sensory modulation dysfunction in children with attention-deficithyperactivity disorder. Dev. Med. Child Neurol. 43, 399-406.

McBurnett, K., and Lahey, B. B. (1994). "Psychophysiological and neuroendocrine correlates of conduct disorder and antisocial behavior in children and adolescents," in Progress in Experimental Personality and Psychopathology Research, eds, D. C. Fowles, P. Sutker and S. H. Goodman (New York: Springer), 199-231.

McIntosh, D. N., Miller, L. J., Shyu, V., and Dunn, W. (1999a). "Overview of the short sensory profile," in ed.W. Dunn, The Sensory Profile: Users Manual (San Antonio, TX: The Psychological Corporation), 59-73.

McIntosh, D. N., Miller, L. J., Shyu, V., and Hagerman, R. J. (1999b). Sensorymodulation disruption, electrodermal responses, and functional behaviors. Dev. Med. Child Neurol. 41, 608-615.

Milich, R., Balentine, A. C., and Lynam, D. R. (2001). ADHD combined type and ADHD predominantly inattentive type are distinct and unrelated disorders. Clin. Psychol. Sci. Pract. 8, 463-488.

Miller, L. J., Anzalone, M. E., Lane, S. J., Cermak, S. A., and Osten, E. T. (2007). Concept evolution in sensory integration: a proposed nosology for diagnosis. Am. J. Occup. Ther. 61, 135-140.

Miller, L. J., McIntosh, D. N., McGrath, J., Shyu, V., Lampe, M., Taylor, A. K., Tassone, F., Neitzel, K., Stackhouse, T., and Hagerman, R. J. (1999). Electrodermal responses to sensory stimuli in individuals with fragile X syndrome. Am. J. Med. Genet. 83, 268-279.

Miller, L. J., Reisman, J. E., McIntosh, D. N., and Simon, J. (2001). "An ecological model of sensory modulation," in Understanding the Nature of Sensory Integration with Diverse Populations, eds, S. Smith Roley, E. Blanche and R. 
C. Schaaf (San Antonio, TX: Therapy Skill Builders), 57-82.

Muris, P., Merckelbach, H., Ollendick, T., King, N., and Bogie, N. (2002). Three traditional and three new childhood anxiety questionnaires: their reliability and validity in a normal adolescent sample. Behav. Res. Ther. 40, 753-772.

Neal, J., Edelmann, R. J., and Glachan, M. (2002). Behavioral inhibition and symptoms of anxiety and depression: is there a specific relationship with social phobia? Br. J. Clin. Psychol. 41, 361-374.

Nigg, J.T. (2006). Temperament and developmental psychopathology. J. Child Psychol. Psychiatry 47, 395-422.

Ognibene, T. C. (2002). Distinguishing Sensory Modulation Dysfunction From Attention-deficit/Hyperactivity Disorder: Sensory Habituation and Response Inhibition Processes. Ann Arbor, MI: ProQuest Information and Learning, pp. 1-125 (ProQuest UMI (UMI No. 3074358).

Parham, D., Cohn, E. S., Spitzer, S., Koomar, J. A., Miller, L. J., Burke, J. P., Brett-Green, B., Mailloux, Z., MayBenson, T. A., Smith-Roley, S., Schaaf, R. C., Schoen, S. A., and Summers, C. A. (2007). Fidelity in sensory integration intervention research. Am. J. Occup. Ther. 61, 216-227.

Parush, S., Sohmer, H., Steinberg, A., and Kaitz, M. (2007). Somatosensory function in boys with ADHD and tactile defensiveness. Physiol. Behav. 90, 553-558.

Pfeiffer, B., Kinnealey, M., Reed, C., and Herzberg, G. (2005). Sensory modulation and affective disorders in children and adolescents with Asperger's disorder. Am. J. Occup. Ther. 59, 335-345.

Pliszka, S. R. (1998). Comorbidity of attention-deficit/hyperactivity disorder with psychiatry disorder: an overview. J. Clin. Psychiatry 59, 50-58.

Pliszka, S. R. (2000). Patterns of psychiatric comorbidity with attentiondeficit/hyperactivity disorder. Child Adolesc. Psychiatr. Clin. North Am. 9, 525-540, vii.

Pliszka, S. R. (2003). Psychiatric comorbidities in children with attention deficit hyperactivity disorder: implications for management. Paediatr. Drugs 5, 741-750.

PsychCorp. (1999). Wechsler Abbreviated Scale of Intelligence Manual (WASI). San Antonio, TX: Harcourt Assessment, Inc.

Reynolds, S., and Lane, S. J. (2007). Diagnostic validity of sensory overresponsivity: a review of the literature and case reports. J. Autism Dev. Disabil. 38, 516-529.

Reynolds, C. R., and Richmond, B. O. (2005). Revised Children's Manifest Anxiety Scale, 9th Edn. Los Angeles: Western Psychological Services.

Reynolds, S., and Lane, S. J. (2009). Sensory over-responsivity and anxiety in children with ADHD. Am J Occup. Ther. 63, 433-440 (available online).

Reynolds, S., Lane, S. J., and Gennings, C. (2009). The moderating role of sensory over-responsivity in HPA activity: a pilot study with children diagnosed with ADHD. J. Atten. Disord. doi: 10.1177/1087054708329906.

Royeen, C. B., and Lane, S. J. (1992). "Tactile dysfunction and related sensory modulation disorders," in Sensory Integration: Theory and Practice, eds A. Fisher, E. Murray and A. Bundy (Philadelphia: F.A. Davis), 108-136.

Schaaf, R. C., Miller, L. J., Seawell, D., and O'Keefe, S. (2003). Children with disturbances in sensory processing: a pilot study examining the role of the parasympathetic nervous system. Am. J. Occup. Ther. 57, 442-449.

Schatz, D. B., and Rostain, A. L. (2006). ADHD with comorbid anxiety. A review of the current literature. J. Atten. Disord. 10, 141-149.

Schmidt, N. A. (1997). Salivary cortisol testing in children. Issues Compr. Pediatr Nurs. 20, 183-190.

Schoen, S., Miller, L. J., and Green, K. E. (2008). Pilot study of the sensory over-responsivity scales: assessment and inventory. Am. J. Occup. Ther. 62, 393-406.

Sonuga-Barke, F.J.S. (2005). Causal models of attention-deficit/hyperactivity disorder: from common simple deficits to multiple developmental pathways. Biol. Psychiatry 57, 1231-1238. van West, D., Claes, S., and Deboutte, D. (2009). Differences in hypothalamicpituitary-adrenal axis functioning among children with ADHD predominantly inattentive and combined types. Eur. Child Adolesc. Psychiatry. doi 10.1007/s00787-009-0011-1.

Venables, P. H., and Christie, M. J. (1980)

"Electrodermal activity," in Techniques in Psychophysiology, eds I. Martin and P. H. Venables (New York: John Wiley \& Sons), 3-68.

Conflict of Interest Statement: The authors declare that the research was conducted in the absence of any commercial or financial relationships that could be construed as a potential conflict of interest.

Received: 01 April 2009; paperpending published: 11 August 2009; accepted: 09 March 2010; published online: 29 March 2010. Citation: Lane SJ, Reynolds $S$ and Thacker L (2010) Sensory over-responsivity and ADHD: differentiating using electrodermal responses, cortisol, and anxiety. Front. Integr. Neurosci. 4:8. doi: 10.3389/fnint.2010.00008

Copyright $\odot 2010$ Lane, Reynolds and Thacker. This is an open-access article subject to an exclusive license agreement between the authors and the Frontiers Research Foundation, which permits unrestricted use, distribution, and reproduction in any medium, provided the original authors and source are credited. 